\title{
Modeling the Luminance Spatial-Angular Distribution in Lighting Scenes*
}

\author{
Vladimir Budak [0000-0003-4750-0160], Victor Chembaev [0000-0002-2488-4184], \\ Tatiana Meshkova [0000-0003-1008-2161] and Victor Zheltov [0000-0001-6768-2518] \\ Department of lighting technology, \\ Moscow Power Engineering Institute, Moscow, Russia \\ budakvp@mpei.ru
}

\begin{abstract}
Now days regulatory documents for non-special lighting systems, the illuminance and various parameters derived from it are normalized as a quantitative characteristic. In most cases, all calculations are carried out for illuminance on the floor of the room or on an imaginary working plane located at the height of the table. However, illuminance is an integral characteristic of incident light, while the human eye responds to light reflected from the surface. That is, if we take a completely black surface with a reflection coefficient equal to zero, then formally you can get the required illuminance on it, while visually we will not see anything, since nothing will be reflected from the surface. In terms of the human eye, luminance must be normalized instead of illuminance. Recently, the calculation and measurement of luminance was an extremely difficult task, so the it is understandable, that current regulatory documents describes almost illuminance normalization, but not luminance. This paper aims to modeling luminance spatial-angular distribution, which enables us to run the assessment of the lighting quality.
\end{abstract}

Keywords: Luminance angle distribution, Quality of lighting, Global illumination.

\section{Introduction}

One of the key areas of lighting engineering is the design of lighting systems (LS). The number of characteristics should be determined during LS designing: the types of lighting devices, their number, location, direction, etc. Now days, the designer is guided by regulatory documents that determine the qualitative and quantitative characteristics of lighting.

In current regulatory documents for non-special LS (office, industrial, commercial and others), the illuminance and various parameters derived from it are normalized as a quantitative characteristic (ratio of minimum to maximum illumination, etc.) In most

Copyright $(\subset 2020$ for this paper by its authors. Use permitted under Creative Commons License Attribution 4.0 International (CC BY 4.0).

* Publication financially supported by RFBR grant №19-01-00435 
cases, all calculations are carried out for illuminance on the floor of the room or on an imaginary working plane located at the height of the table. However, illuminance is an integral characteristic of incident light, while the human eye responds to light reflected from the surface. That is, if we take a completely black surface with a reflection coefficient equal to zero, then formally you can get the required illuminance on it, while visually we will not see anything, since nothing will be reflected from the surface. In terms of the human eye, luminance must be normalized instead of illuminance. Recently, the calculation and measurement of luminance was an extremely difficult task, so the it is understandable, that current regulatory documents describes almost illuminance normalization, but not luminance.

Introduction of Unified Glare Rating (UGR), as one of possible quality indicators for non-specialized LS, can partially eliminate this problem:

$$
U G R=8 \lg \left[\frac{0,25}{L_{a}} \sum_{i=1}^{N} \frac{L_{i}^{2} \omega_{i}}{p_{i}^{2}}\right],
$$

where $L_{i}$-glare light source luminance, $\mathrm{cd} / \mathrm{m}^{2}, \omega_{i}$-glare light source angular size, steradian, $p_{i}$ - light source position index relative to line of sight, $L_{a}$ - adaptation luminance, $\mathrm{cd} / \mathrm{m}^{2}$.

Thus, UGR allowed to express the quality of lighting with just one number and was accepted into regulatory documents. Today the designer is guided by illuminance as a quantitative characteristic and UGR as a qualitative assessment of lighting, when designing non-special OS, such as retail, office, public, industrial premises and many others, where UGR can answers the question - how comfortable will it be for a person within the lighting installation.

However, the formula is valid only for small-angle uniform gloss sources. UGR cannot take into account extended uneven glare. The matter is complicated by the fact that the DIALux and Relux simulation programs widely used in lighting design are based on the finite element method and do not solve the global lighting equation with respect to brightness, but the radiosity equation in the diffuse approximation. Which obviously leads to the fact that secondary glare cannot be taken into account. A significant step forward in lighting design is the introduction of DIALux Evo based on the method of Photon maps. However, the methodology for calculating UGR has not changed.

Thus, LS are designed only approximately assessing how comfortable it will be for a person in it and normalizing an invisible characteristic - illuminance. However, at the beginning of the last century, it was suggested that the spatial-angular distribution of luminance plays a key role in the comfort perception [1].

\section{Luminance spatial-angular distribution}

The modeling of lighting installations is based on the well-known in computer graphics global lighting equation, first obtained by James T Kajiya in 1986 [2]

$$
L(\mathbf{r}, \hat{\mathbf{l}})=L_{0}(\mathbf{r}, \hat{\mathbf{l}})+\frac{1}{\pi} \int L\left(\mathbf{r}, \hat{\mathbf{l}}^{\prime}\right) \sigma\left(\mathbf{r} ; \hat{\mathbf{l}}, \hat{\mathbf{I}}^{\prime}\right)\left|\left(\hat{\mathbf{N}}, \hat{\mathbf{I}}^{\prime}\right)\right| \hat{\mathbf{l}}^{\prime},
$$

where $L(\mathbf{r}, \hat{\mathbf{l}})$ - luminance at point $\mathbf{r}$ in direction $\hat{\mathbf{l}}, \sigma\left(\mathbf{r} ; \hat{\mathbf{l}}, \hat{\mathbf{l}}^{\prime}\right)-$ bidirectional reflection 
function (reflection or transmission), $L_{0}$ - direct luminance component, directly from sources, $\hat{\mathbf{N}}-$ normal at point $\mathbf{r}$ to the scene surface element.

The equation is written relative to the point $\mathbf{r}$ located on the surface of the scene, however, in the task of assessing the quality of lighting, the observer is in the volume of the scene. After a series of transformations, one can obtain an equation written with respect to a point in the volume of the scene [3]

$$
\begin{aligned}
& L_{V}(\mathbf{r}, \hat{\mathbf{l}})=L_{0}\left(\mathbf{r}_{\Sigma}, \hat{\mathbf{l}}\right)+\frac{1}{\pi} C_{01} \int L\left(\mathbf{r}_{1}, \hat{\mathbf{l}}^{\prime}\right) \sigma\left(\mathbf{r}_{\Sigma} ; \hat{\mathbf{l}}^{\prime}, \hat{\mathbf{l}}\right) G\left(\mathbf{r}_{1}, \mathbf{r}_{\Sigma}\right) \times \\
& \quad \times \delta\left(\Pi\left(\mathbf{r}-\left|\mathbf{r}-\mathbf{r}_{\Sigma}\right| \hat{\mathbf{l}}\right)\right) \delta\left(\hat{\mathbf{l}}-\frac{\mathbf{r}-\mathbf{r}^{\prime}}{\left|\mathbf{r}-\mathbf{r}^{\prime}\right|}\right) d^{3} r_{1} \frac{d^{3} r_{\Sigma}}{\left(\mathbf{r}-\mathbf{r}_{\Sigma}\right)^{2}},
\end{aligned}
$$

where $G\left(\mathbf{r}_{1}, \mathbf{r}_{\Sigma}\right)$ - equation kernel.

Equation (3) describes the luminance spatial-angular distribution (LSAD) at each point in the scene space. It allows us to approach the question of assessing the quality of lighting not only based on the assessment of individual glare light sources as in UGR, but based on the analysis of a continuous spatial-angular distribution of luminance.

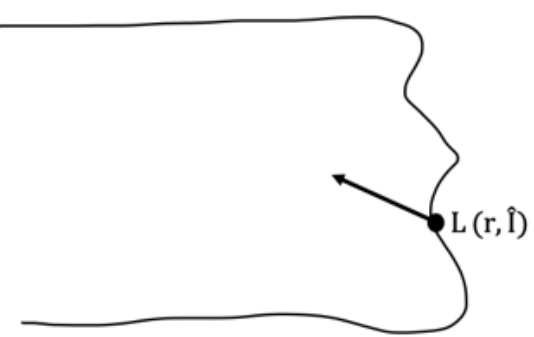

a)

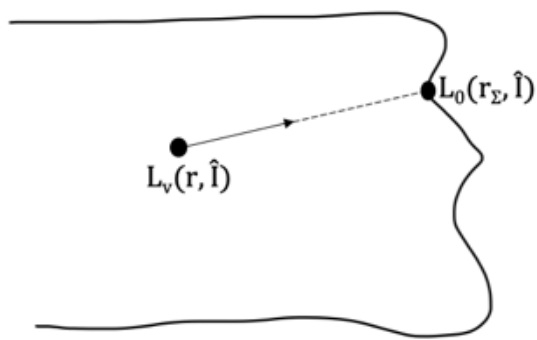

b)

Figure 1. Figure a) shows a point on the surface of the scene, figure b) a point in the volume of the scene at which we are determining the luminance in a given direction

In 1997, the work "Instant Radiosity" was published [4]. This publication laid the foundation for a new approach to solving the global illumination equation, which ultimately did not become widespread in computer graphics. The author formulated the algorithm of the new method in a phenomenological approach, without giving its full mathematical justification, using only disparate formulas to describe individual parts of the modeling process.

In our work, we propose applying Local estimation method of the Monte Carlo method to solving the global illumination equation.

The solution of the global illumination equation (2) can be expanded in the Neumann series [5]

$$
\begin{gathered}
L(\mathbf{r}, \hat{\mathbf{l}})=L_{0}(\mathbf{r}, \hat{\mathbf{l}})+\frac{1}{\pi} \int L_{0}\left(\mathbf{r}_{1}, \hat{\mathbf{l}}_{1}\right) \sigma\left(\mathbf{r} ; \hat{\mathbf{l}}_{1}, \hat{\mathbf{l}}\right) G\left(\mathbf{r}_{1}, \mathbf{r}\right) d^{3} r_{1}+ \\
+\frac{1}{\pi} \int \frac{1}{\pi} \int L_{0}\left(\mathbf{r}_{1}, \hat{\mathbf{l}}_{1}\right) \sigma\left(\mathbf{r}_{2} ; \hat{\mathbf{l}}_{1}, \hat{\mathbf{l}}_{2}\right) G\left(\mathbf{r}_{1}, \mathbf{r}_{2}\right) d^{3} r_{1} \sigma\left(\mathbf{r} ; \hat{\mathbf{l}}_{2}, \hat{\mathbf{l}}\right) G\left(\mathbf{r}_{2}, \mathbf{r}\right) d^{3} r_{1}+\ldots
\end{gathered}
$$


After a series of transformations, expression (4) can be interpreted as a Markov chain with a transition probability determined by the kernel of the equation

$$
k\left(x_{i} \rightarrow x\right)=\frac{\sigma\left(\mathbf{r} ; \hat{\mathbf{l}}_{i}, \hat{\mathbf{l}}\right) G\left(\mathbf{r}_{i}, \mathbf{r}\right)}{p_{2}\left(x_{i} \rightarrow x\right)} .
$$

As a result of the construction of the Markov chain, we can evaluate the luminance at a given point in a given direction on the surface of the scene. Such estimation can be called a Local estimation of the Monte Carlo method, since it allows you to directly calculate the luminance at a given point on the scene surface in a given direction.

We cannot construct a similar scheme for the global illumination equation for a point located in the volume of the scene (3). However, we have to determine the quality of lighting based on the spatial-angular distribution of luminance - so, it is necessary to be able to obtain an angular distribution of luminance for an arbitrary point in the scene. Two additional $\delta$-functions and appear in the equation, $\delta\left(\Pi\left(\mathbf{r}-\left|\mathbf{r}-\mathbf{r}_{\Sigma}\right| \hat{\mathbf{l}}\right)\right) \quad$ и $\delta\left(\hat{\mathbf{i}}-\frac{\mathbf{r}-\mathbf{r}^{\prime}}{\left|\mathbf{r}-\mathbf{r}^{\prime}\right|}\right)$, which depend on the desired direction $\hat{\imath}$. This makes it impossible to directly simulate the equation. When modeling the brightness at a given point and direction of the scene space $(\mathbf{r}, \hat{\mathbf{l}})$, we cannot get into the direction $\hat{\mathbf{l}}$ we need from the nodes of the trajectory of the Markov chain. To do this, we need one more additional node fixing the intermediate point $\mathbf{r}_{\Sigma}$ of the equation (3). This approach is called Double Local estimation [5]. A Double Local estimation allows modeling the global illumination equation for a point in the scene in space and thereby obtain a spatialangular distribution of luminance.

\section{Lighting quality}

\subsection{Lighting quality criterion based on LSAD}

Today, when designing non-specialized LS, such as office premises, public places, shops, shopping centers, etc., in fact, there is only one criterion that describes the quality of the LS - the Unified Glare Rating (UGR). The discomfort in the luminance spatial-angular distribution is affected not only by the absolute value of the observed luminance, but also by the ratio of the source-background luminance difference to the background luminance (adaptation brightness) known as contrast [6]. The ratio of contrast to threshold contrast can serve as a criterion for lighting quality. In the case of a continuous spatial-angular distribution over the lighting scene, a natural generalization of contrast is the ratio of the gradient of the distribution of luminance over the observation field to the average over the luminance field [7][8]. Increasing the gradient value, the boundary between the glossy source and the background will become more defined, and the quality of lighting, accordingly, will decrease. That is, the larger the source and the higher the luminance gradient around the bright source, the greater the contribution to the discomfort of this source. Note that in real life, extended glare are both sources of discomfort and contribute to the luminance of the 
LSAD adaptation. The generalized contrast at the scene point can be determined [9]:

$$
K(x, y)=\frac{|\operatorname{grad}(L(x, y) p(x, y))|}{\bar{L}},
$$

where

$$
\bar{L}=\frac{1}{A} \int_{(A)} L(x, y) p(x, y) d x d y, \quad A=\int_{(A)} d x d y,
$$

$x, y$-coordinates of the point on the projection of the scene, $L$ - luminance of a given point in the direction of observation, $\bar{L}$ - field-average luminance, $p(x, y)$-some weight function, taking into account the different contribution to the reaction of the eyes of points located in the center of the field of view and on the periphery. In the criterion $p$ formula, it carries the same physical meaning as the position index in the UGR formula. Thus, we can formulate the lighting quality criterion $Q$ as the field-weighted contrast $K(x, y)$, assigned to a certain threshold:

$$
Q=\frac{1}{K_{t h r}} \int K(x, y) d x d y,
$$

where $K_{t h r}$ - contrast threshold value.

It is assumed that the threshold contrast will be determined by the light engineering problem.

\subsection{Determination of threshold luminance}

It is obvious that in the proposed form, any subtle change of luminance will contribute to the quality criterion, since there will be a change in the luminance gradient. It is also obvious that changes in contrasts in luminances below a certain limit will not make a real contribution. So, for example, if there is a direct source of light in the field of view or a glare from it in the room, contrasts in the dark corner most likely will not play a role in the perception of lighting quality. But if we formally carry out the calculation, then they will give a contribution. Thus, contrasts below a certain threshold $L_{t h r}$ should not actually be taken into account.

In 1946, Blackwell conducted an enormous amount of research to establish threshold contrasts in solving the detection problem [10]. In the experiment, the relationship between the threshold contrast and the luminance of adaptation for various angular sizes of the target was established. Figure 2. graphs of this dependence are presented. 


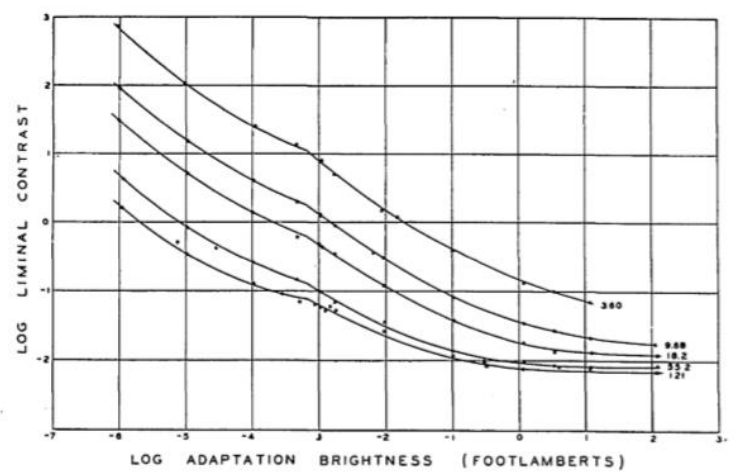

Figure 2. Dependence of the logarithm of the threshold contrast on the logarithm of the brightness of adaptation for 5 angular sizes: 121.0, 55.2, 18.2, 9.68, 3.60 angular minutes.

Based on this study, a number of thresholds can be considered as $L_{t h r}$. By setting the minimum size of the element that you want to detect - for example, it can be the size of the character when reading text or signage from a certain distance. Then, knowing the brightness of the adaptation, which can be taken as the average brightness over the field, can be determined from the results of Blackwell's research $\Delta L$ - a threshold change in brightness to solve the detection problem. Then, by setting a certain number of threshold excesses, one can already determine the threshold brightness as

$$
L_{t h r}=N \Delta L \text {, }
$$

where $N$ - is a certain number, depending on the difference between the really solved problem and the threshold.

So, as the threshold brightness of the cutoff of contrasts that do not affect the quality criterion, we take a certain number of thresholds, for example, $10 \Delta L_{t h r}$.

Then the expression for contrast can be written as

$$
K(x, y)=\left\{\begin{array}{llc}
L(x, y) \leq L_{t h r} & \rightarrow & 0 \\
L(x, y)>L_{t h r} & \rightarrow & K(x, y)
\end{array} .\right.
$$

As part of our work, an experiment was conducted to determine the quality of lighting at stations of the Moscow Metro. Figure 3 shows a scatter map of the average observer rating from the lighting quality criterion. A linear correlation coefficient was also calculated between the average rating of observers and the values of the quality criterion, which amounted to 0.61 . The correlation coefficient can reach 1 , in this case all points must lie strictly on the diagonal. To interpret the value of the correlation coefficient, you can use the Cheddock scale. 


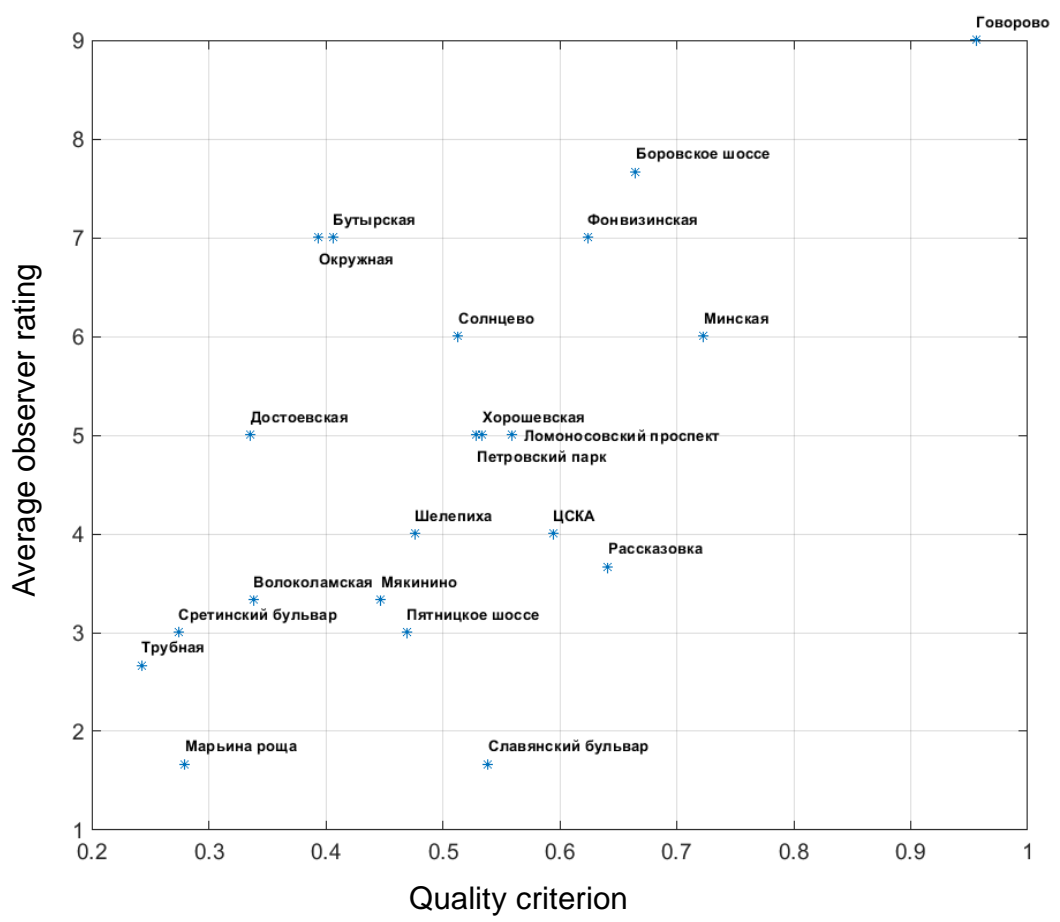

Figure 3. The scattering of the average observer rating and the lighting quality criterion with an adaptation luminance equal to the average luminance at the station

As part of the work, we examined the effect of the number of thresholds $\mathrm{N}$ on the correlation coefficient. Figure 4 shows a graph of the correlation coefficient of the results on the number of thresholds.

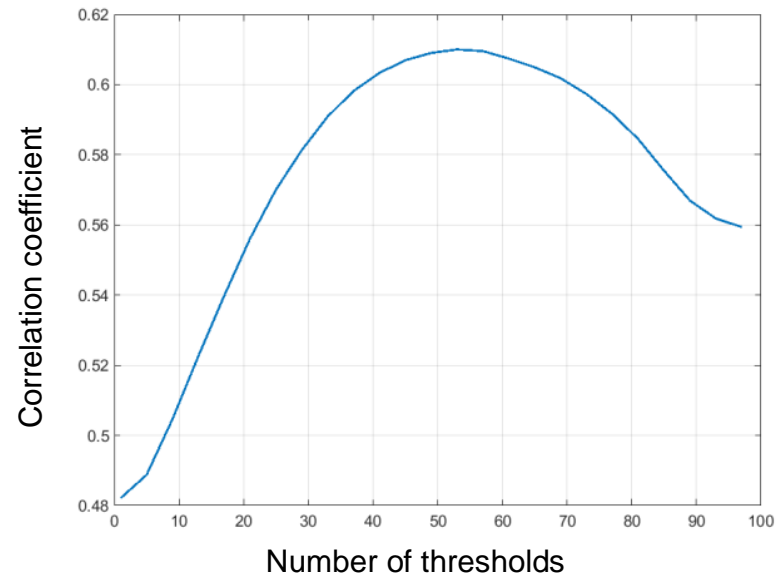

Figure 4 The dependence of the correlation coefficient on the number of thresholds 


\section{Conclusion}

From studies back in 1915, it is known that the luminance spatial-angular distribution significantly affects the quality of illumination perceived by the observer. Based on LSAD, it is possible to assess the quality characteristics of lighting, including mainly normalized today, Unified Glare Rating UGR. However, until recently, there were neither mathematical methods, nor computational capabilities to model exactly LSAD. Thus, the existing regulatory documents and tools of engineers by the lighting designer are built around radiosity modeling.

Today we have a situation when, from the point of view of theory, mathematical solution methods, algorithms, computational capabilities, physical measurement tools, lighting engineering is ready to switch to the analysis of a characteristic that is truly perceived by the human eye - luminance. And as a consequence of this process, it is possible to change the design paradigm from design to specified quantitative characteristics, to design taking into account the quality of lighting based on the LSAD.

\section{References}

1. Ferree, C., Rand, G.: The efficiency of the eye under different conditions of lighting. Trans. Illum. Eng. Soc. IES, v.10, 407-447 (1915).

2. Kajiya, J.: The rendering equation. In: SIGGRAPH Proceedings of the $13^{\text {th }}$ Computer Graphics Proceedings, vol.20, N4, pp. 143-150. Association for Computing Machinery, New York, NY, United States (1986).

3. Budak, V., Zheltov, V., Notfulin, R., Chembaev, V.: Relation of instant radiosity method with local estimations of Monte Carlo method. $24^{\text {th }}$ International Conference in Central Europe on Computer Graphics, Visualization and Computer Vision on Journal of WSCG, pp. 189-196. University of West Bohemia, Czech Republic (2016).

4. Keller, A.: Instant radiosity. In: SIGGRAPH Proceedings of the 24th annual conference on Computer graphics and interactive techniques, pp. 49-56. Association for Computing Machinery, New York, NY, United States (1997).

5. Marchuk, G., Mikhailov, G., Nazareliev, M., Darbinjan, R., Kargin, B., Elepov, B.: MonteCarlo Methods in Atmospheric Optics. Springer-Verlag, Berlin (1980).

6. Scheir, G., Hanselaer, P., Bracke, P., Deconinck, G., Ryckaert, W.: Calculation of the Unified Glare Rating based on luminance maps for uniform and non-uniform light soruces. Building and Environment, 84, pp. 60-67 (2015).

7. Budak, V., Zheltov, V., Lubenchenko, A., Freidlin, K., Shagalov, O.: Fast and accurate algorithm for the numerical simulation of radiative transfer in turbid media. Optika atmosfery i okeana, vol.26, pp. 739-746. Institute of Atmospheric Optics, Tomsk (2016).

8. Budak, V., Zheltov, V., Meshkova, T., Notfullin, R..: Evaluation of illumination quality based on spatial-angular luminance distribution. Light \& Engineering, 4(25), 24-31(2017).

9. Budak, V., Zheltov, V., Meshkova, T., Chembaev, V.: Experemental study of the new criterion of lighting quality based on analysis of luminance distribution at Moscow metro stations. Light \& Engineering 3(28), 98-105 (2020).

10. Blackwell, H.: Contrast Thresholds of the Human Eye. Josa, 11(36), 624-643 (1946). 